\title{
Validation of a Tn5 transposon mutagenesis system for Gluconacetobacter diazotrophicus through characterization of a flagellar mutant
}

Luc F. M. Rouws • Jean L. Simões-Araújo • Adriana S. Hemerly · José I. Baldani

Published online: 11 January 2008

(C) Springer-Verlag 2008

Erratum to: Arch Microbiol

DOI 10.1007/s00203-007-0330-x

In Materials and methods, the phrase

"PVU-RIGHT (GAAAAACAGCATTCCAGGTATTA GA)" should be substituted with "PVU-RIGHT (CAAAAG TTTATGCATTTCTTTCCAG)".

The online version of the original article can be found under doi:10.1007/s00203-007-0330-x.

L. F. M. Rouws · J. L. Simões-Araújo · J. I. Baldani ( $\square)$ CNPAB/EMBRAPA, BR465, Km7,

Seropédica, RJ 23890-000, Brazil

e-mail: ibaldani@cnpab.embrapa.br

L. F. M. Rouws · A. S. Hemerly Instituto de Bioquímica Médica, UFRJ,

Rio de Janeiro, RJ 21941-590, Brazil

\section{A. S. Hemerly}

Laboratório de Biologia Molecular de Plantas,

Instituto de Pesquisas do Jardim Botânico do Rio de Janeiro,

Rua Pacheco Leão 915, Rio de Janeiro, RJ 22460-030, Brazil 